\title{
Nodal induced by hypoxia exposure contributes to dacarbazine resistance and the maintenance of stemness in melanoma cancer stem-like cells
}

\author{
HONG LI ${ }^{1,2}$, JUNJIE CHEN $^{1}$, XIAO WANG $^{2}$, MEI HE $^{2}$, ZHENYU ZHANG $^{1}$ and YING CEN ${ }^{1}$ \\ ${ }^{1}$ Department of Burns and Plastic Surgery Unit, West China Hospital of Sichuan University, Chengdu, Sichuan 610041; \\ ${ }^{2}$ Department of Burns and Plastic Surgery, The Second People's Hospital of Chengdu, Chengdu, Sichuan 610017, P.R. China
}

Received November 8, 2017; Accepted April 17, 2018

DOI: $10.3892 /$ or.2018.6387

\begin{abstract}
Nodal signaling has a critical role in the processes of embryogenesis and is necessary for maintaining cell stemness. However, its upregulation in melanoma is positively correlated with malignant potential, including cancer progression, metastasis and recurrence, and a recent report has revealed its role in promoting self-renewal capacity in melanoma. Our study aimed to explore the effects of hypoxia exposure, which is one of the main causes of chemoresistance in melanoma, on the physiological processes of melanoma cancer stem-like cells (CSCs) via regulating Nodal. A375 CSCs were enriched by culturing in serum-free medium (SFM) and were analyzed for the expression levels of Nodal and its correlated proteins by semi-quantitative western blotting. Lentiviral-packaged Nodal coding sequence or short-hairpin RNA (shRNA) was employed. After hypoxia exposure, the effects on glucose uptake, ATP production and $\mathrm{O}_{2}$ consumption were detected, and whether Nodal contributed to the proliferation, invasion, colony formation, self-renewal capacity and chemoresistance was evaluated. We demonstrated that hypoxia exposure induced Nodal expression and activated the $\operatorname{Smad} 2 / 3$ pathway in A375 CSCs. Hypoxic-induced Nodal partially promoted dacarbazine resistance, promoted invasion and self-renewal capacity, but not proliferation which was further confirmed using Nodal knockdown. Blockage of Nodal signaling activity with the small-molecule inhibitor SB431542 partially reversed Nodal-induced chemoresistance. Nodal knockdown further sensitized A375 CSCs to dacarbazine after SB431542 pretreatment, indicating the involvement of proNodal in dacarbazine resistance. The introduction of mut-proNodal induced chemoresistance further confirming the role of proNodal in
\end{abstract}

Correspondence to: Professor Ying Cen, Department of Burns and Plastic Surgery Unit, West China Hospital of Sichuan University, 37 Guoxue Road, Wuhou, Chengdu, Sichuan 610041, P.R. China E-mail: cy2000cyhuaxi@163.com

Key words: cancer stem-like cells, CSCs, Nodal, dacarbazine, chemoresistance, hypoxia this process. Taken together, our results demonstrated that Nodal induced by hypoxia exposure induced a malignant phenotype and chemoresistance in A375 CSCs, and proNodal also contributed to these processes, indicating that Nodal may be a potential therapeutic target for melanoma.

\section{Introduction}

Melanoma is one of the most aggressive types of skin cancer, and is the most deadly cutaneous neoplasm (1). In Western populations, although it accounts for less than $5 \%$ of all skin cancers, melanoma is one of the most common cancers among young adults and is one of the most common causes of death $(2,3)$. Although melanoma is relatively rare, the increasing incidence over the past two decades has raised a major health concern, and once metastasis to distant organ occurs, the prognosis becomes extremely poor. Metastatic melanoma is resistant to many types of chemotherapeutic agents (4). To date, dacarbazine is considered to be the reference single agent for the management of advanced melanoma, and dacarbazine treatment exhibits effective responses in $\sim 15-25 \%$ of patients (4). However, in metastatic melanoma patients, the 6-year median survival rate is extremely low (5).

The embryonic protein Nodal, belongs to the transforming growth factor (TGF)- $\beta$ family, and plays critical roles in maintaining the self-renewal capacity and pluripotency of human embryonic stem cells (hESCs) $(6,7)$. Emerging evidence has revealed its involvement in promoting the growth and progression of various types of cancer, including melanoma, glioma, breast, prostate and endometrial cancer (8-12). In both melanoma and its CSC subpopulation, accounting for $0.5 \%$ of melanoma cancer cells, activated Nodal/Activin signaling promotes tumorigenicity and maintains self-renewal capacity (13). In most cases, Nodal induces signal transduction after being cleaved by the proprotein convertases Furin or Pace4 to remove an N-terminal prodomain from precursor Nodal (proNodal) (14). A recent report also revealed that proNodal, but not mature Nodal, induces Nodal signaling transduction (15). However, it is still unknown which forms of Nodal, proNodal or mature Nodal regulates CSC properties.

Hypoxia, as one of the factors critical in the biological processes of CSCs, plays crucial roles in the activation of 
pathways involved in the maintenance of CSC functions $(16,17)$, and is implicated in the development of chemoresistance. It was recently uncovered that hypoxia exposure converted non-cancer stem cells to CSCs in the melanoma cancer cell line A375 (18). Furthermore, Quail et al found that, in melanoma and breast cancer cells, hypoxia induced Nodal expression and caused promotion of cell invasion and angiogenic phenotypes, suggesting that Nodal may also be regulated by hypoxia in pancreatic CSCs (19).

In the present study, we investigated the effect of hypoxia exposure on Nodal expression and CSC properties in melanoma cancer cells. Our study revealed that hypoxia-induced Nodal enhanced CSC properties, including self-renewal capacity, sphere forming ability and chemoresistance. Moreover, both proNodal and mature Nodal induced by hypoxia exposure contributed to a higher tumorigenic potential.

\section{Materials and methods}

Cell culture, sphere formation and treatments. Human melanoma cancer cell line, A375, was purchased from the American Type Culture Collection (ATCC; Manassas, VA, USA) and cultured in Dulbecco's modified Eagle's medium (DMEM) supplemented with $10 \%$ fetal bovine serum (FBS), 100 units $/ \mathrm{ml}$ penicillin and $100 \mu \mathrm{g} / \mathrm{ml}$ streptomycin (Life Technologies; Thermo Fisher Scientific, Inc., Waltham, MA, USA).

For sphere formation, $2 \times 10^{5}$ cells were seeded in each 6-well plate and cultured with DMEM-F12 (1:1) medium containing human basic fibroblast growth factor (bFGF; 10 ng/ml; PeproTech, Rocky Hill, NJ, USA), human epidermal growth factor (EGF; $20 \mathrm{ng} / \mathrm{ml}$; PeproTech) and 2\% B27 (Life Technologies; Thermo Fisher Scientific, Inc.). Every three days, the medium was half-refreshed.

Hypoxic cultures were carried out in a humidified hypoxia workstation Invivo ${ }_{2} 400$ model (Baker Ruskinn Technology Ltd., Bridgend, UK). Briefly, medium was pre-exposed to $20 \%$ $\mathrm{O}_{2}, 5 \% \mathrm{O}_{2}, 2 \% \mathrm{O}_{2}, 1 \% \mathrm{O}_{2}$, or $0.5 \% \mathrm{O}_{2}$ balanced with nitrogen and $5 \% \mathrm{CO}_{2}$. Then, the cells were cultured in pre-treated medium and maintained in corresponding conditions for 24 or $48 \mathrm{~h}$.

Western blotting. Cells were lysed with Laemmli sample buffer (50 mM Tris $\mathrm{pH} 6.8,1.25 \%$ SDS, $10 \%$ glycerol) and heated at $100^{\circ} \mathrm{C}$ for $15 \mathrm{~min}$ for denaturalization. SDS-PAGE electrophoresis (6-12\% gradient) was performed to fractionate total protein. Blots were then incubated with the following primary antibodies purchased from Abcam: CD44 (cat. no. ab157107), CD133 (cat. no. ab19898), Nanog (cat. no. ab21624), Sox2 (cat. no. ab97959), $\beta$-actin (cat. no. ab8226), cleaved Nodal (cat. no. ab81287), proNodal (cat. no. ab109317), ALK4 (cat. no. ab109300), ALK7 (cat. no. ab111121), Smad2/3 (cat. no. ab202445), p-Smad2 (cat. no. ab53100), p-Smad3 (cat. no. ab52903), HK-II (cat. no. ab104832), Glut-I (cat. no. ab115730), PDK-1 (cat. no. 110025) and HIF-1 $\alpha$ (cat. no. ab113642). Primary antibodies were diluted at 1:1,000 in PBS and incubated with the blotted membrane at room temperature for $2 \mathrm{~h}$. After three washes with PBS supplemented with $0.1 \%$ Tween-20 at room temperature, goat anti-mouse secondary antibody (cat. no. ab97040) (for $\beta$-actin, PDK-1 and HIF-1 $\alpha$ ) was used; for other antibodies, goat anti-rabbit secondary antibody (cat. no. ab7090) was used. $\beta$-actin was used as a loading control. Proteins were detected using ECL (Life Technologies; Thermo Fisher Scientific, Inc.) and visualized on X-ray film (Kodak, Japan).

Lentivirus production and cell infection. We obtained lentivirus-based shRNA constructs targeting human Nodal (shNodal; 5'-CCGGGCGGT TTC AGATGG ACCTATTCTCGAGAATAGGTCCATCTGAAACCGCTTT TTG-3') (LV-shNodal) as well as a control shRNA-expressing plasmid (shScrambled; 5'-CCGGCTATGGACGCTCTT ATGTACTGGCGCCAGTACATAAGAGCGTCCATAGTTT TTG-3') (LV-shScrambled). The oligonucleotides were cloned into the shRNA-pGCL-GFP lentiviral vector (Shanghai Genchem, Shanghai, China), respectively. Infectious viruses were produced by contransfecting the lentiviral vectors and packaging constructs (pHelper1.0 and pHelper2.0) into 293T cells using Lipofectamine ${ }^{\mathrm{TM}}$ reagent (Life Technologies; Thermo Fisher Scientific, Inc.).

The lentiviruses containing the Nodal coding sequences (LV-Nodal) for overexpressing Nodal were produced by Genomeditech Company (Shanghai, China), which were labeled with RFP. Lentiviruses containing the empty vector (LV-vector) were used as a negative control. Cells were transduced with the packaged lentiviruses at a multiplicity of infection (MOI) of 1:10 (cell:virus) (20) and 72-h later, the transduced cells were subsequently collected for further analysis.

Glucose uptake assay. For measuring glucose uptake, $1 \times 10^{6}$ cells were plated in 6-well plates overnight for attaching. The cells were incubated in glucose-free medium for $30 \mathrm{~min}$ at $37^{\circ} \mathrm{C}$ in a $\mathrm{CO}_{2}$ incubator and then $2-\left[{ }^{3} \mathrm{H}\right]$ deoxyglucose at $1 \mu \mathrm{Ci}$ was added into the medium for a 2-h incubation. The cells were washed with ice-cold PBS three times, and transferred to scintillation vials for counting as described previously $(21,22)$.

Lactate measurements. Cells $\left(1 \times 10^{6}\right)$ were plated in 6-well plates overnight and the medium was replaced with serum-free medium for $12 \mathrm{~h}$. The supernatant was collected and diluted with PBS. For measuring lactate, the EnzyChrom ${ }^{\mathrm{TM}}$ L-Lactate assay kit (BioAssay Systems, Hayward, CA, USA) was employed following the manufacturer's instruction and was measured at $565 \mathrm{~nm}$ using a microplate reader (Synergy 2 Multi-Mode Microplate Reader; BioTek, Winooski, VT, USA).

ATP production. Cells $\left(1 \times 10^{6}\right)$ were plated in 6-well plates and allowed to adhere overnight. ATP Lite assay kit (PerkinElmer, Inc., Waltham, MA, USA) was employed to measure ATP production following the manufacturer's instruction.

Reactive oxygen species (ROS) detection. Total intracellular ROS was assessed by flow cytometry using the dichlorofluorescein (DCF) oxidation assay. The intracellular ROS oxidizes cleaved DCFH-DA which enters into cells. Target cells $\left(5 \times 10^{5}\right)$ were incubated with DCFH-DA $(10 \mu \mathrm{M})$ for $1 \mathrm{~h}$ at $37^{\circ} \mathrm{C}$, followed by 3 washes with ice-cold PBS, and ROS fluorescence was analyzed using 3 laser Navios flow cytometers (Beckman Coulter, Brea, CA, USA) with green fluorescence channel (FL1). 
Cell death analysis. Cells $\left(5 \times 10^{4}\right)$ were collected, washed in PBS and stained with $1 \mu \mathrm{g} / \mathrm{ml}$ PI for $30 \mathrm{~min}$ in darkness. The stained cells were washed with ice-cold PBS for three times and analyzed by flow cytometry using 3 laser Navios flow cytometers. PI-positive cells were regarded as dead cells, and the percentage of dead cells was determined.

Cell viability assay. Cells were grown in 96-well plates, and viable cell numbers were determined with the CellTiter-Glo Luminescent Cell Viability Assay kit (Promega, Madison, WI, USA) following the manufacturer's guide.

Transwell cell invasion assays. Matrigel (Millipore Corp., Darmstadt, Germany) was diluted 1:2 in DMEM/F-12 and $60 \mu \mathrm{l}$ of diluted Matrigel was added to the upper chamber of Transwell plates. Then, the chambers were incubated at $37^{\circ} \mathrm{C}$ in a $5 \% \mathrm{CO}_{2}$ incubator for $2 \mathrm{~h}$. Single-cells were resuspended in DMEM/F-12 and seeded into the upper chamber at a density of $2 \times 10^{4}$ cells/well. Subsequently, $600 \mu \mathrm{l}$ of DMEM/F-12 supplemented with $20 \mathrm{ng} / \mathrm{ml} \mathrm{EGF,} 10 \mathrm{ng} / \mathrm{ml} \mathrm{bFGF}$ and $2 \%$ B27 was added to the lower chamber. After a 24-h incubation, the inserts were collected, and the cells on the lower surface were fixed in $4 \%$ paraformaldehyde and stained with crystal violet for $15 \mathrm{~min}$. The cells in five random views were counted under a X71 (U-RFL-T) fluorescence microscope (Olympus, Melville, NY, USA).

Cloning of cleavage-resistant Nodal precursor (mut-proNodal). The expression vector for mut-proNodal has been described (23) and the lentiviral vector containing mut-proNodal coding sequence ( $\mathrm{LV}$-mut-proNodal) was packaged as previously described (23).

Serial replating assay. Cells were replated at a clonal density (2,000 cells/well) and cultured in serum-free medium supplemented with $2 \%$ B-27, $10 \mathrm{ng} / \mathrm{ml}$ EGF and $20 \mathrm{ng} / \mathrm{ml} \mathrm{bFGF}$. The medium was half-replaced every 3 days. After 10 days, PBS-washed cells were fixed with $4 \%$ paraformaldehyde and stained with $0.1 \%$ crystal violet for $10 \mathrm{~min}$ and washed again with PBS, and the colonies were counted. For replating, the same amount of cells was plated in serum-free medium. After 10 days, the same procedure was performed three times.

Statistical analysis. All data were normalized to control values for each assay and are presented as mean \pm standard deviation (SD). Multigroup comparisons of the means were carried out by one-way analysis of variance (ANOVA) test with post hoc contrasts by Student-Newman-Keuls test. Significance was chosen as $\mathrm{P}<0.05$.

\section{Results}

Hypoxia upregulates the expression of Nodal protein in A375 CSCs. Serum-free medium culturing was used for enriching the stem-like cells from A375 cells in this study. After 14 days of culturing, spheres were completely formed and showed morphological similarity with embryonic tissues (Fig. 1A). In order to characterize the stem-like characteristics of the A375 subpopulation enriched using SFM culturing, we examined the expression levels of several stem cell markers (CD133, CD44,
OCT4, Nanog and SOX2) and found that all were upregulated in the A375 CSCs compared to levels in the A375 cells at both the mRNA and protein levels (Fig. 1B and C). We further used in vitro serial replating assay to examine the self-renewal capacity of the A375 CSCs and the results confirmed the stem-like property (Fig. 1D). Nodal is reported to be restricted to embryonic tissues and hESCs, and re-emerges during tumorigenesis in several types of cancers (24). This promoted us to assess the differential expression of Nodal in A375 and A375 CSCs. Unexpectedly, compared to A375, no detectable difference in Nodal was found in the A375 CSCs (Fig. 1E).

For detecting the regulatory factor of tumor progression, hypoxia, which is an important environmental change in many cancers, was employed to determine its effect on the regulation of Nodal protein expression. We exposed A375 CSCs to varying levels of $\mathrm{O}_{2}$ for 24 and $48 \mathrm{~h}$. Uncleaved Nodal protein (uncleaved NODAL, uNODAL, $39 \mathrm{kDa}$ ) and cleaved and secreted Nodal protein (cleaved NODAL, cNODAL, $15 \mathrm{kDa}$ ) were detected in whole cell lysate and in concentrated medium by ultrafiltration individually. The data showed that exposure of A375 CSCs to varying $\mathrm{O}_{2}$ concentrations $\left(0.5-20 \% \mathrm{O}_{2}\right)$ for 24 and $48 \mathrm{~h}$ upregulated the Nodal protein level at concentrations $\leq 2 \% \mathrm{O}_{2}$ (Fig. 1F). We also detected the expression pattern of Nodal receptor, ALK4/7, and no detectable changes were observed (Fig. 1F). Surprisingly, hypoxic exposure undetectably disturbed Nodal mRNA levels, indicating its post-transcriptional regulation on Nodal (data not shown). We then investigated whether Nodal/Nodal receptors are coupled to Smad signaling pathway by detecting phosphorylated Smad2/3 in A375 CSCs. Western blot analysis showed that Smad2, but not Smad3, was phosphorylated in A375 CSCs exposed to hypoxia at oxygen concentration of $0.5 \%$ and co-incubated with $100 \mathrm{ng} / \mathrm{ml} \mathrm{rhNodal}$ (as a positive control) (Fig. 1G).

Hypoxic-induced Nodal enhances glucose uptake and promotes glycolysis. Nodal protein expression was reported to be positively correlated with hexokinase (HK)-II, glucose transporter (Glut)-1 and pyruvate dehydrogenase kinase (PDK)-1 by activating hypoxia-inducible factor (HIF)-1 $\alpha$, which is induced by hypoxic exposure and directly regulates energy metabolism (25-27). Firstly, by introducing Nodal, we confirmed the positive correlation of Nodal with HK-II, Glut-1, PDK-1 and HIF-1 $\alpha$ in A375 CSCs (Fig. 2A), and hypoxia exposure exerted similar effects. For ascertaining whether hypoxia-induced Nodal contributes to the upregulation of HIF-1 $\alpha$, HK-II, Glut-1 and PDK-1, in hypoxia-induced A375 CSCs transfected with Nodal shRNA, semi-quantitative western blotting was performed. As shown in Fig. 2B, hypoxia exposure markedly upregulated all these proteins, and Nodal knockdown detectably attenuated their protein levels. In hypoxic-exposed A375 CSCs, knockdown of Nodal significantly decreased 2-[ $\left.{ }^{3} \mathrm{H}\right]$ deoxyglucose uptake (Fig. 2C) and lactate accumulation (Fig. 2D). We next assessed whether Nodal contributes to the regulation of ATP production. As expected, Nodal knockdown negatively affected ATP production (Fig. 2E).

Nodal expression prevents excess ROS production and apoptosis in A375 CSCs. It has been reported that promotion 
A

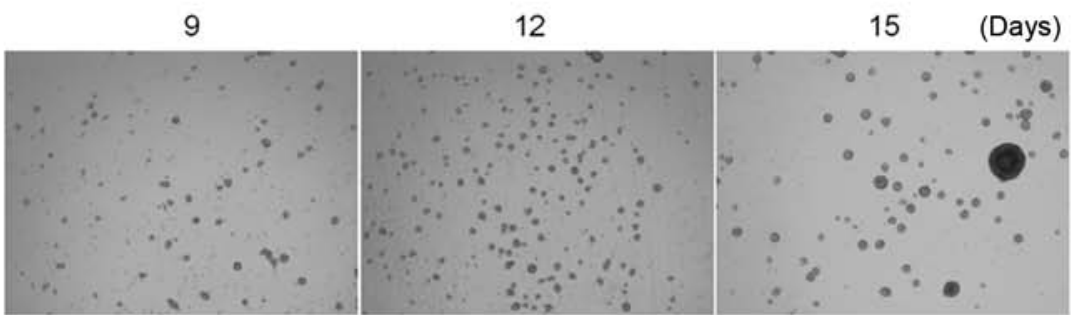

C

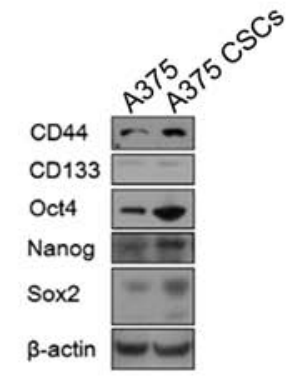

D

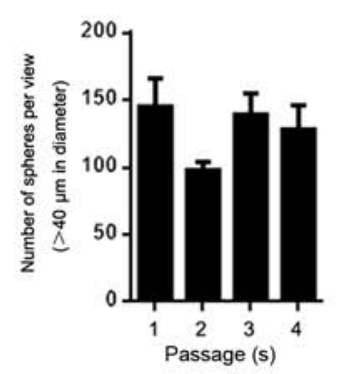

$\mathrm{F}$

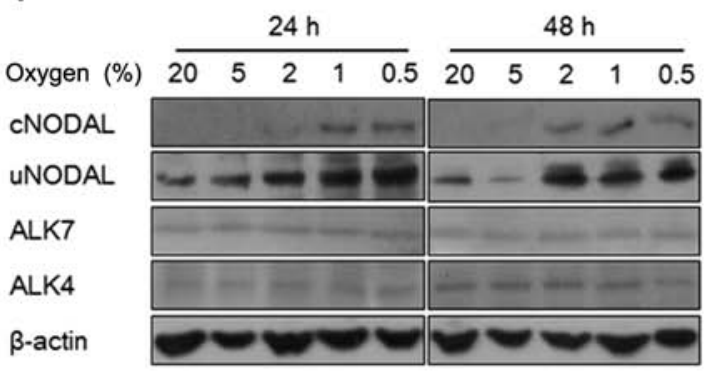

$E$

B

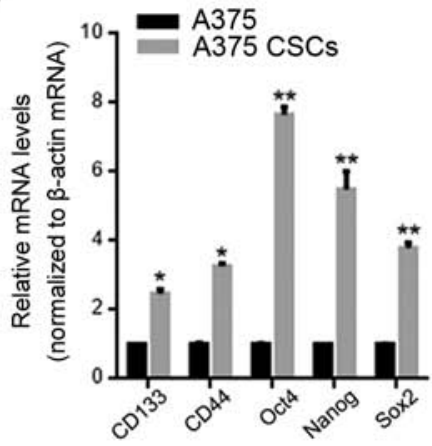

Figure 1. Hypoxia exposure stimulates Nodal expression and activates Nodal signaling. (A) A375 cells were cultured in SFM supplemented with cytokines for 9-15 days. qPCR (B) and semi-quantitative western blot (C) analyses of (CSC) markers in A375 and A375 CSCs. ${ }^{*} \mathrm{P}<0.05$, ${ }^{* *} \mathrm{P}<0.01 ; \mathrm{P}$-values were obtained against A375 cells. (D) Serial replating assay for analyzing the self-renewal capacity of A375 CSCs. (E) Relative Nodal mRNA (left panel) and protein (right panel) levels. (F) Expression levels of uncleaved Nodal (uNODAL), cleaved Nodal (cNODAL), ALK4 and ALK7 were semi-quantified after exposure to different concentrations of oxygen. (G) Activation of Nodal signaling by hypoxia exposure as detecting by levels of Smad2/3, p-Smad2 and p-Smad3; SFM, serum-free medium; CSC, cancer stem cell.

of glycolysis prevents excess ROS production (28). Thus, we examined whether Nodal expression regulates ROS production. As shown in Fig. 3A, hypoxia exposure promoted ROS production and knockdown of Nodal markedly decreased accumulated ROS, which was confirmed quantitatively (Fig. 3B). As ROS is important for hypoxia-induced cell death, we analyzed whether Nodal modulates ROS-induced cell death in A375 CSCs. Knockdown of Nodal expression sensitized A375 CSCs against hypoxia-induced cell death, whereas the addition of ROS scavenger, $\mathrm{N}$-acetyl-cysteine (NAC) slightly abolished the effect of Nodal, suggesting that Nodal enhances ROS detoxification mechanisms (Fig. 3C).

Hypoxic-induced Nodal partially contributes to decarbazine resistance in A375 CSCs. For investigating the roles of Nodal in A375 CSCs, we produced lentiviral particles coding for shRNA targeting the Nodal mRNA (LV-shNodal and LV-scrambled) and for overexpressing Nodal mRNA (LV-Nodal and LV-vector). The infectious efficacy and Nodal protein levels were detected (Fig. 4A and B). A375 CSCs were infected with LV-Nodal, LV-shNodal, or LV-Nodal/LV-shNodal to determine their $\mathrm{IC}_{50}$ value for dacarbazine, respectively. As expected, Nodal induced by hypoxic exposure and overexpressed by lentiviral infection desensitized A375 CSCs to dacarbazine, and knockdown of Nodal by LV-shNodal sensitized A375 CSCs to dacarbazine, indicating the critical role of Nodal protein in dacarbazine response (Fig. 4C). For further confirm the necessity of Nodal on dacarbazine desensitization, A375 CSCs were infected by LV-Nodal or LV-shNodal at MOI 10, 25, 50 and 100 to determine their $\mathrm{IC}_{50}$. With the decrease in Nodal protein, A375 CSCs presented more sensitivity to dacarbazine (Fig. 4D). However, in hypoxic-exposed A375 CSCs, upregulation of Nodal protein caused no detectable changes in the sensitization to dacarbazine, possibly because of its high endogenous level (data not shown). We then blocked the Nodal signal by SB 431542 treatment in A375 CSCs to ascertain whether uncleaved precursor (proNodal) is involved in dacarbazine response. Surprisingly, after 
A

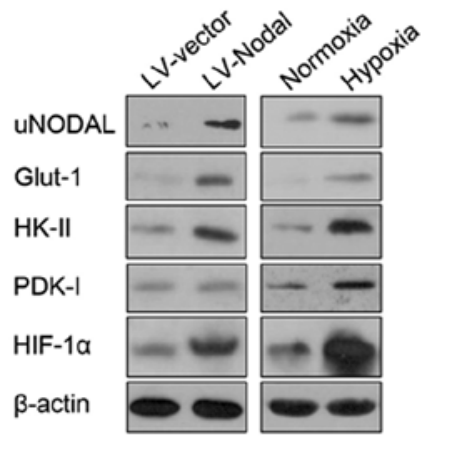

D
B

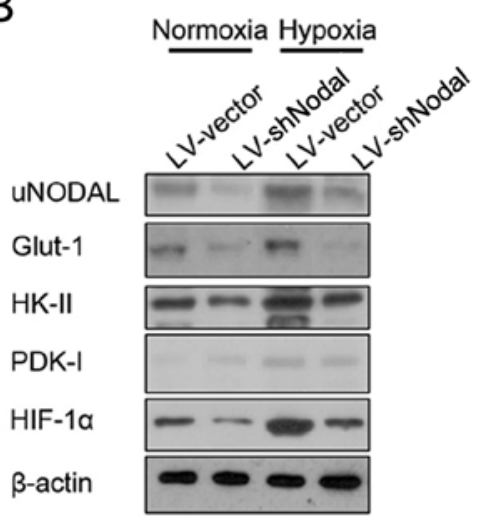

E

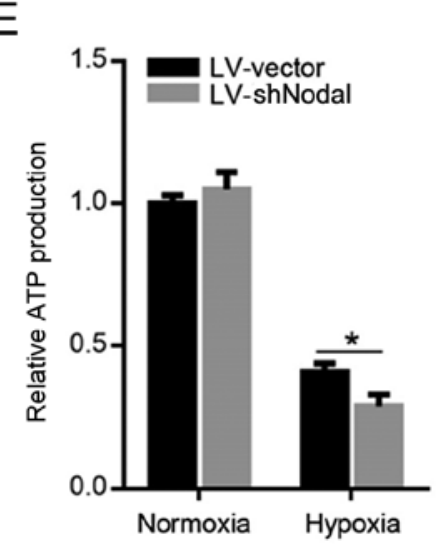

C

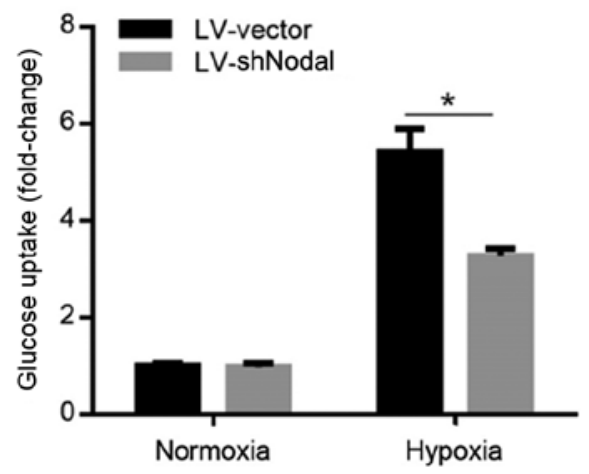

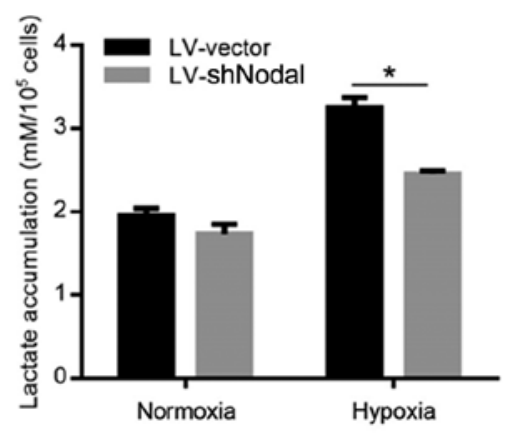

Figure 2. Hypoxic-induced Nodal promotes glycolysis. (A) Semi-quantitative western blotting was performed to detect uncleaved Nodal (uNODAL), Glut-1, HK-II, PDK-I and HIF-1 $\alpha$. (B) After knockdown of Nodal, the expression of uNODAL, Glut-1, HK-II, PDK-I and HIF-1 $\alpha$ were detected. In hypoxic-induced A375 CSCs, glucose uptake (C) lactate accumulation (D) and ATP production relative level normalized to LV-vector group exposed to normoxia (E) were detected. ${ }^{*} \mathrm{P}<0.05$.

A

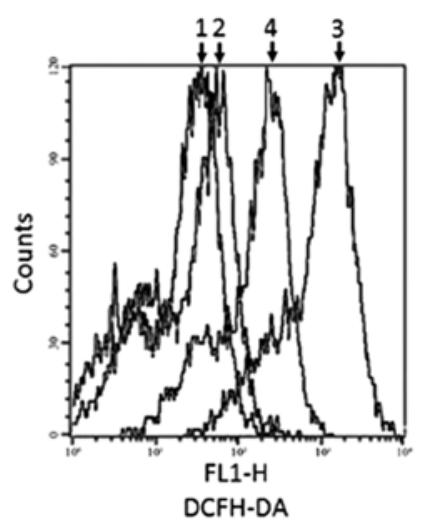

B

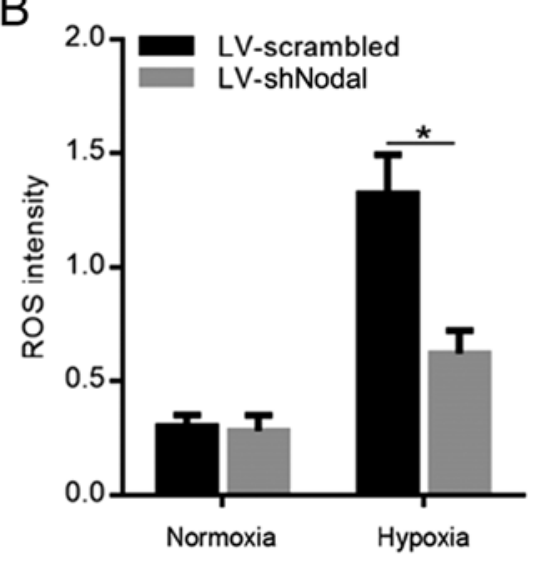

C

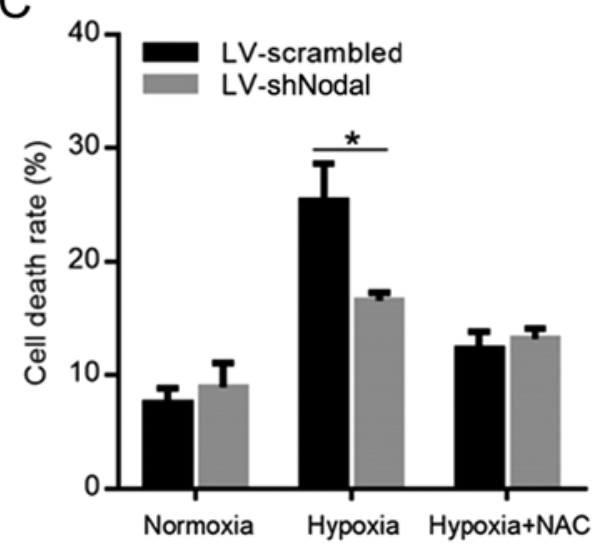

Figure 3. Nodal exerts protective effects on A375 CSCs via preventing excess ROS accumulation. (A) ROS levels in cells treated with (spike 1) normoxia+LV-scrambled; (spike 2) normoxia+LV-shNodal; (spike 3) hypoxia+LV-scrambled; (spike 4) hypoxia+LV-shNodal were detected separately. (B) ROS levels were quantitatively measured. (C) Percentage of cell death was determined. " $\mathrm{P}<0.05$. CSCs, cancer stem cells; ROS, reactive oxygen species.

SB431542 blockage, knockdown of Nodal still sensitized hypoxic-exposed A375 CSCs to dacarbazine, indicating that proNodal also contributes to dacarbazine resistance in an undetermined manner (Fig. 4E).

Hypoxic-induced Nodal promotes cell invasion and maintains cell stemness, but not proliferation in A375 CSCs. Because of its tight association with malignancies in cancer and CSCs $(29,30)$, we assessed the effects of hypoxic-induced Nodal on proliferation, invasion and preservation of stemness. Hypoxia exposure slightly affected the distribution of cell cycle phases and cell viability (data not shown), possibly due to high endogenous Nodal protein level. Then, the invasiveness affected by hypoxia exposure was detected, and the 
A

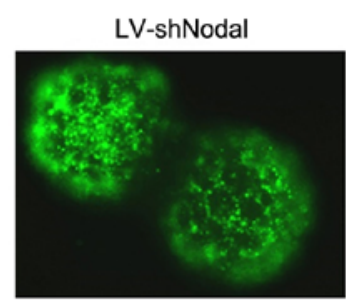

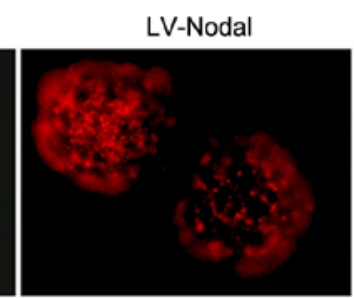

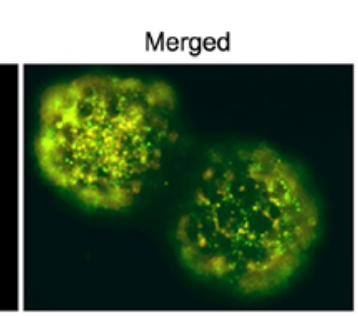

B

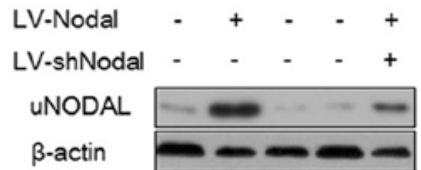

C
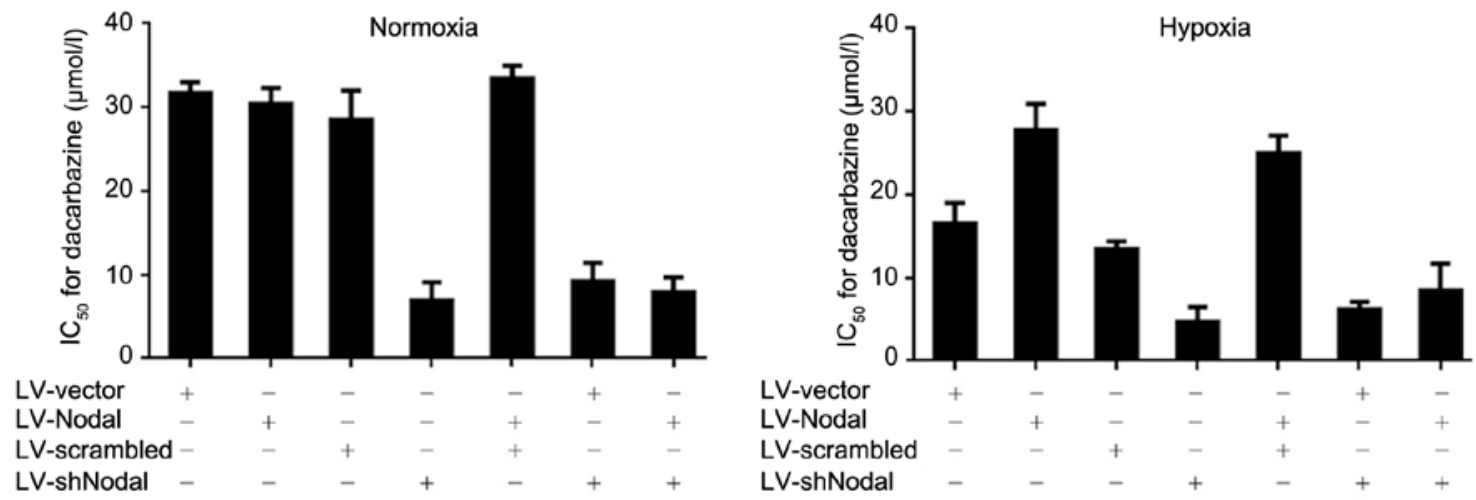

D

Normoxia
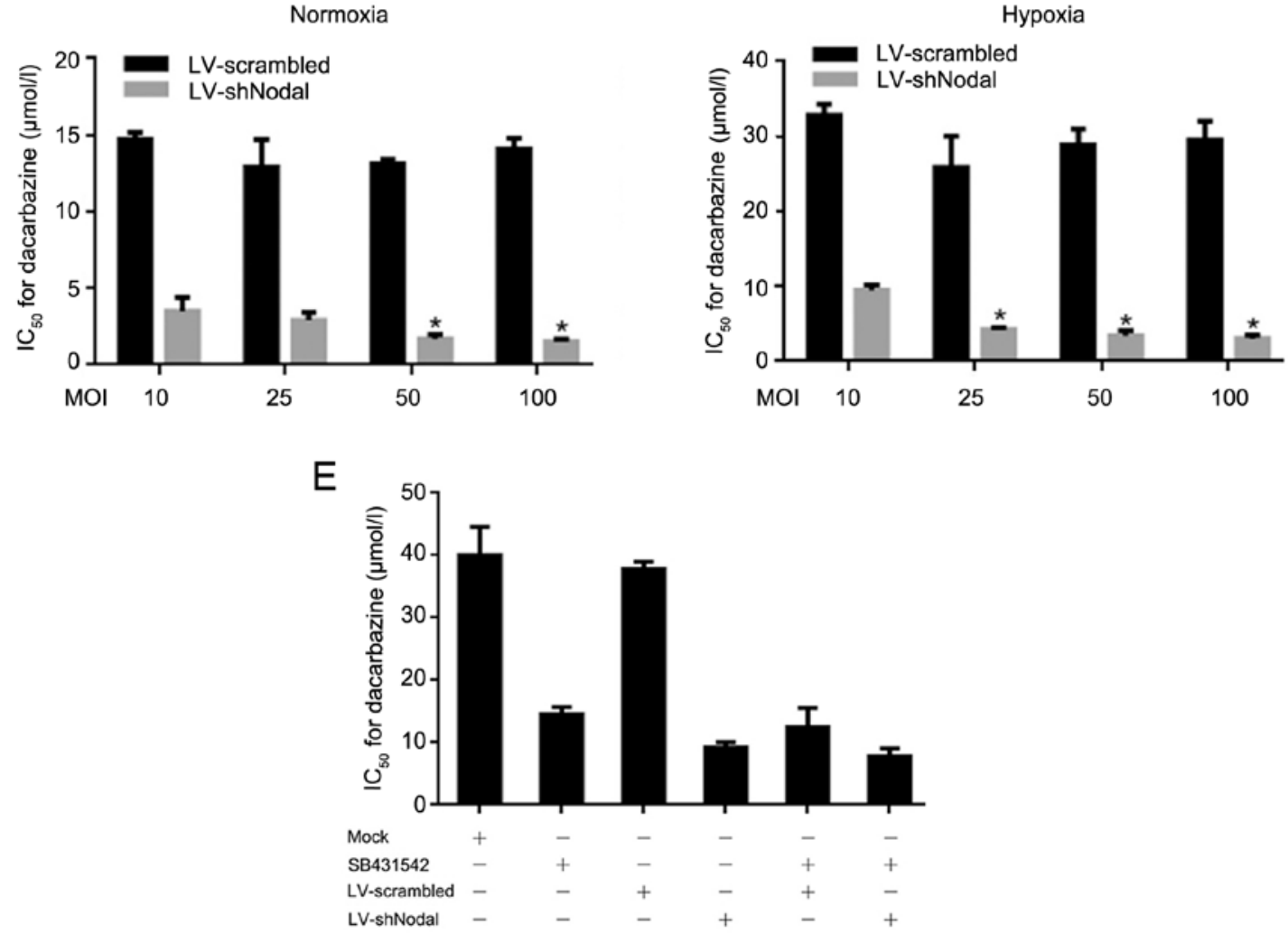

Figure 4. Nodal induced by hypoxia exposure desensitizes A375 CSCs to dacarbazine. (A) Confirmation of infectious efficacy of lentiviral vector for shRNA targeting to Nodal mRNA (LV-shNodal) or overexpressing Nodal (LV-Nodal). (B) Western blot analysis was performed to further confirm the level of uncleaved Nodal (uNODAL) intracellularly. (C) Evaluation of $\mathrm{IC}_{50}$ in normoxia or hypoxia exposed A375 CSCs. (D) Nodal induced by hypoxia desensitized A375 CSCs to dacarbazine depending on Nodal protein levels. (E) Dacarbazine sensitivity is regulated by both Nodal expression and Nodal signaling inhibitor, SB431542. $\mathrm{P}<0.05$. CSCs, cancer stem cells.

results showed that hypoxia exposure promoted invasion via upregulation of Nodal in A375 CSCs (Fig. 5A). For evaluating the capacity of self-renewal properties in A375 CSCs, colony formation and sphere formation assays were employed. The colony formation results demonstrated that both knockdown of Nodal expression and blockage by SB431542 in A375 CSCs apparently reduced colony formation (Fig. 5B). To further characterize the effects of hypoxia-induced Nodal on A375 
A
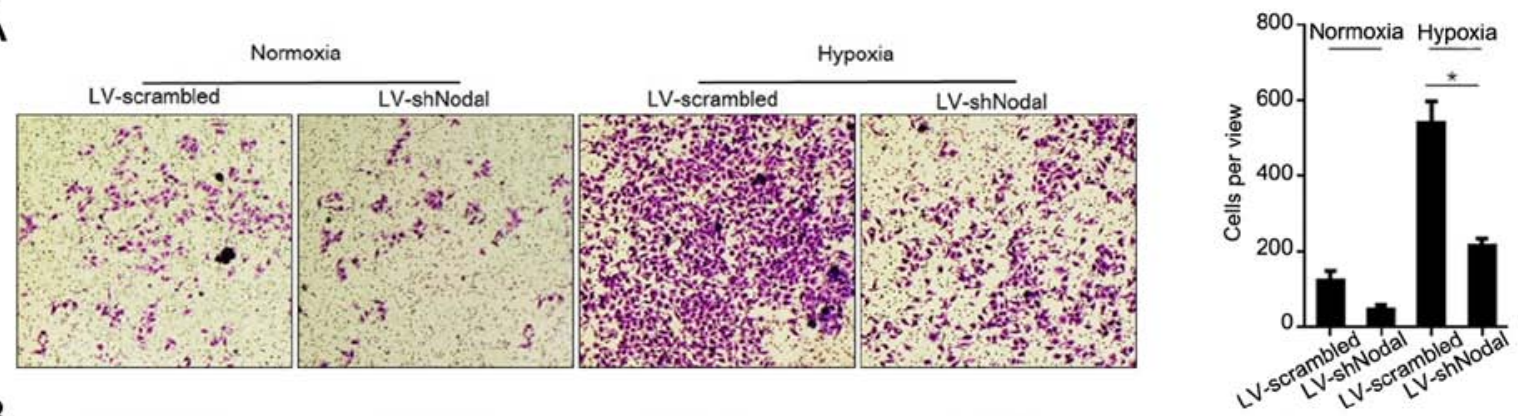

B

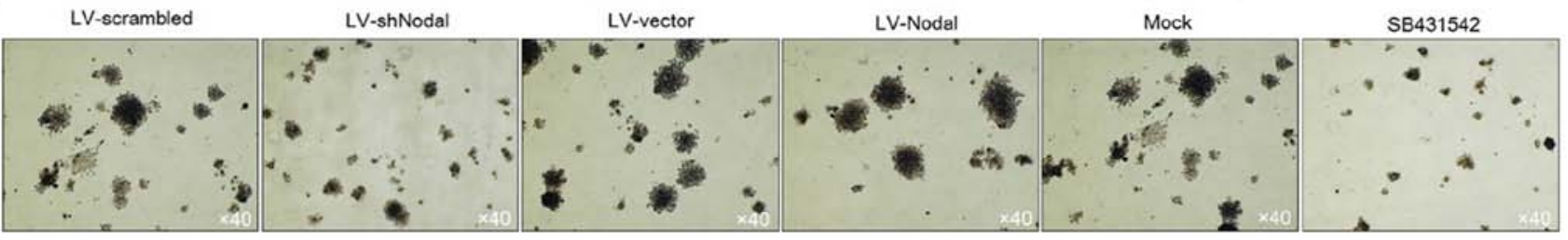

C

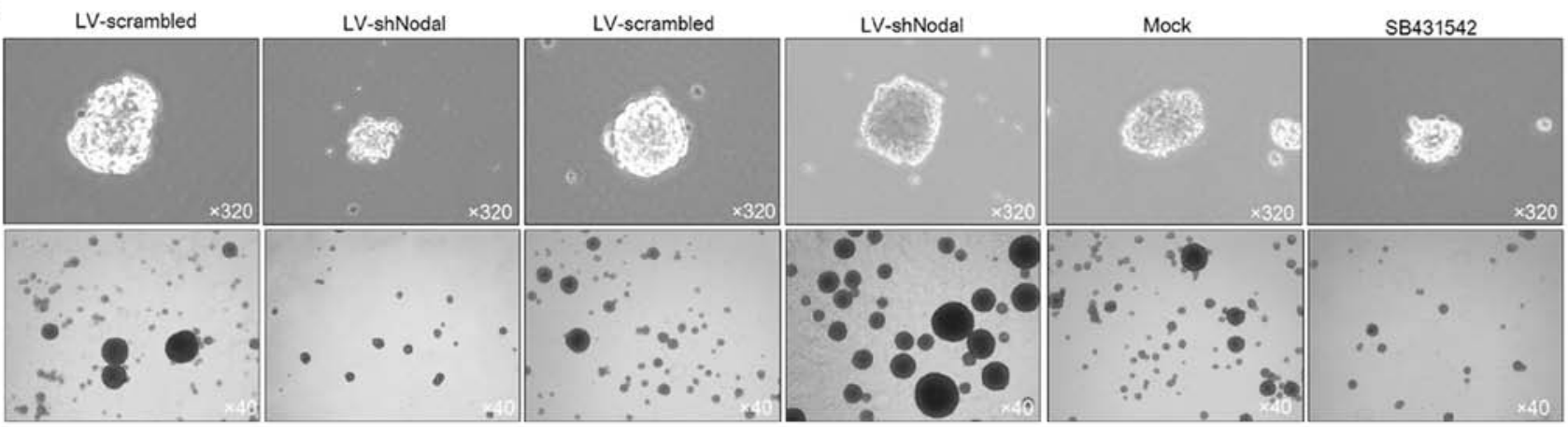

D

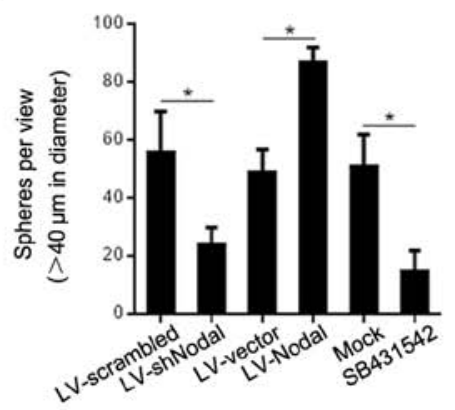

E

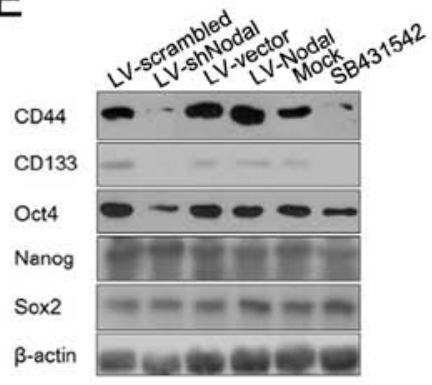

Figure 5. Nodal induced by hypoxia promotes cell invasion and maintains CSC stemness. (A) Transwell assay was performed to detect invasive ability in cells after Nodal is knocked down (left panel) and cells were counted in five random views (right panel). (B) Colony formation and (C) sphere formation were analyzed in A375 CSCs with different expression levels of Nodal. (D) Sphere formation was quantified by sphere counting of spheres ( $>40 \mu \mathrm{m})$ in five random views. (E) Semi-quantitative western blot analysis was performed to detect markers of stemness, including CD44, CD133, Oct4, Nanog and Sox 2 . ${ }^{\mathrm{P}}<0.05$. CSC, cancer stem cell.

CSCs, we overexpressed Nodal by lentiviral infection to simulate upregulation of hypoxic-induced Nodal, and to determine the sphere formation ability. Similarly, upregulated Nodal expression promoted the formation of spheres in the A375 CSCs in SFM (Fig. 5C). Simultaneously, both knockdown of Nodal expression and blockage by SB431542 in A375 CSCs caused dissociation of spheres (Fig. 4C). We counting the spheres $\geq 40 \mu \mathrm{m}$ in diameter, and found that Nodal expression promoted the sphere forming rate, and Nodal knockdown and SB431542 exposure diminished the sphere forming rate (Fig. 5D). By considering the regulatory roles of Nodal on stemness maintenance, we next evaluated its effects on related genes, including CD44, CD133, Oct4, Nanog, and
Sox2. Without disturbing Nanog and Sox 2 levels, expression of Nodal was positively correlated with CD44, CD133 and Oct4 (Fig. 5E), which was consistent with its effect on spheroid morphology.

Both cleaved Nodal and proNodal contribute to dacarbazine resistance in A375 CSCs. Nodal is secreted as a precursor protein by endoproteolytic cleavage. It has been revealed that proNodal displays a subset of activities at physiological concentrations (31-33). By considering the potential involvement of proNodal in physiological processes of A375 CSCs, we investigated whether the precursor and/or cleaved Nodal contribute to dacarbazine resistance. We first examined 

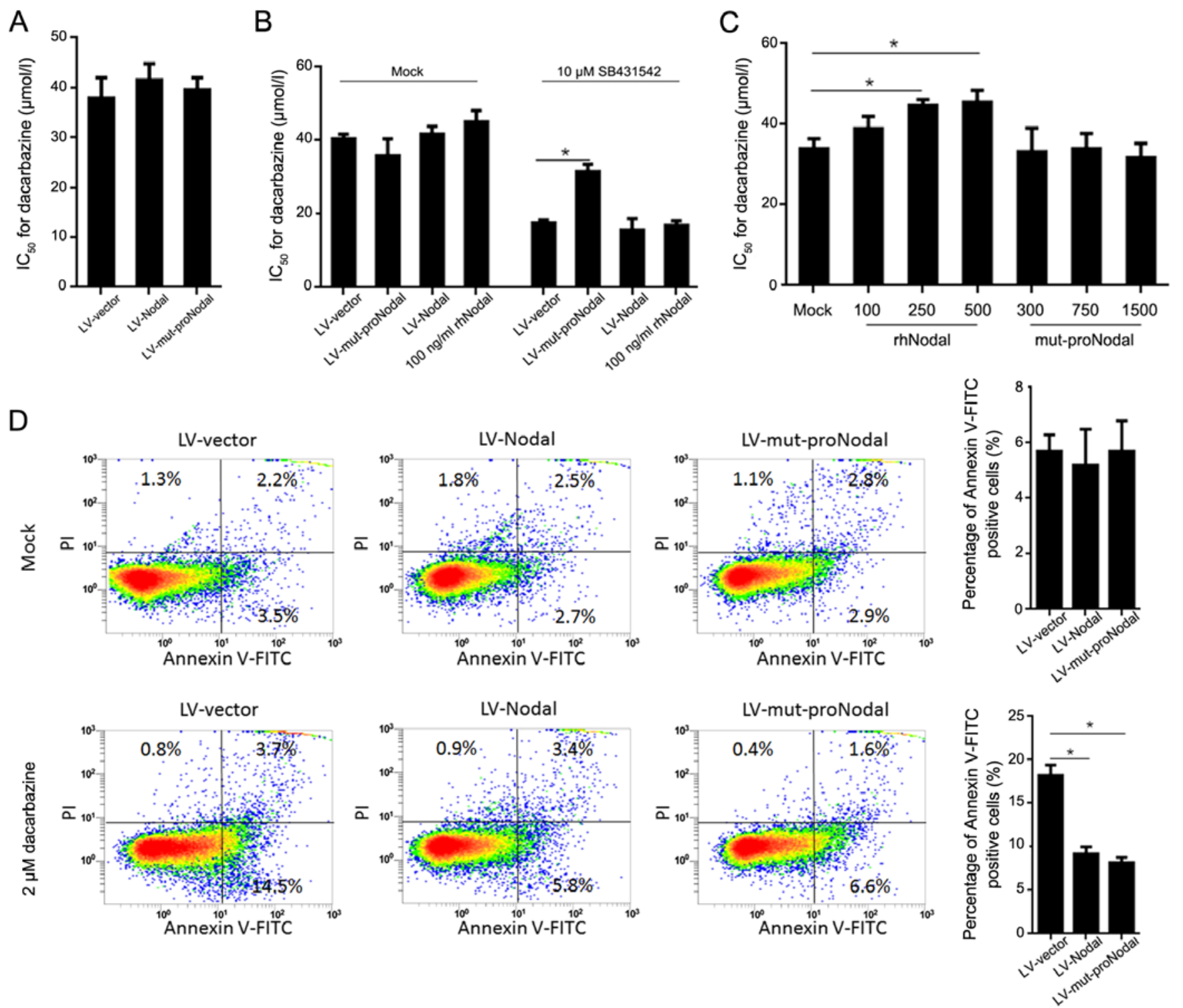

Figure 6. Cleaved Nodal and proNodal desensitize A375 CSCs to dacarbazine. (A and B) Dacarbazine sensitivities were analyzed in A375 CSCs infected with LV-vector, LV-Nodal or LV-proNodal, respectively, with or without SB431542 pretreatment. (C) Effects of rhNodal or mut-proNodal protein on gemcitabine sensitivity via ALK4/7 receptor were detected by co-incubation. (D) Annexin V-FITC/PI staining was performed to detect the apoptotic rate with or without $2 \mu \mathrm{M}$ dacarbazine treatment. ${ }^{*} \mathrm{P}<0.05$. CSCs, cancer stem cells.

whether a recombinant Nodal cleavage mutant (mut-proNodal) which is resistant to specific cleavage can regulate dacarbazine sensitization in A375 CSCs. In A375 CSCs infected with LV-mut-proNodal, dacarbazine resistance was not disturbed, and SB431542-pretreated A375 CSCs were desensitized to dacarbazine after LV-mut-proNodal infection (Fig. 6A and B). For ascertaining whether mut-proNodal functions via recognizing the Nodal receptor, we exposed A375 CSCs to purified mut-proNodal and found that, rhNodal but not mut-proNodal exposure desensitized A375 CSCs to dacarbazine and was reversed by SB431542 co-exposure (Fig. 6C). For further confirming the apoptosis induced by dacarbazine treatment, Annexin V-PI staining was analyzed by flow cytometry. The results showed that, after $2 \mu \mathrm{M}$ dacarbazine treatment for $24 \mathrm{~h}$, introduction of mut-proNodal significantly reduced the apoptotic rate, with a robustness similar to that observed for LV-Nodal (Fig. 6D). Unexpectedly, introduction of mutant-proNodal failed to regulate self-renewal capacity and sphere formation (data not shown), indicating that proNodal might function via an independent undetermined manner.

\section{Discussion}

In the present study, we report that hypoxia exposure of A375 CSCs enhanced the CSC phenotype, tumor formation, invasion and chemoresistance. Hypoxia exposure upregulated Nodal protein and subsequently activated downstream signaling pathways. Blockage of Nodal pathway using ALK-4/5/7 inhibitor (SB431542; $10 \mu \mathrm{M}$ ) inhibited the CSC properties of hypoxia-exposed A375 CSCs. Moreover, it was revealed that hypoxic-induced Nodal enhanced glucose uptake and promoted glycolysis, and subsequently exerted protective effects on A375 CSCs via preventing ROS accumulation. 
Nodal plays essential roles in the processes of embryonic development and maintains the pluripotent properties of stem cells during embryogenesis $(34,35)$. Generally, Nodal expression is specifically expressed in embryonic tissues and hESCs and is undetectable in most highly differentiated tissues. However, it is found to be upregulated and contributes to increasing cancer cell aggressiveness, tumorigenicity and metastasis in several types of cancer, including melanoma, breast cancer and melanoma (36-38). These findings indicate that the abnormal expression of Nodal promotes cancer cell proliferation, invasion and migration and inhibits apoptosis. It was reported that hypoxia exposure induces Nodal expression predominantly mediated via an HIF- $1 \alpha$-dependent pathway in melanoma cells. However, the effect of hypoxia exposure on melanoma CSCs is still unknown. In the present study, to further explore the regulatory effect of hypoxia exposure on Nodal expression and CSC properties in A375 CSCs, we exposed A375 CSCs to hypoxia and found that Nodal was induced after hypoxia exposure. Overexpression of Nodal increased the number and size of spheroids and promoted colony formation. In addition, semi-quantitative western blot analysis revealed that induced Nodal protein resulted in increased expression of CD44, CD133 and Oct4. For confirming whether induced Nodal expression by hypoxia exposure was responsible for these changes in expression, Nodal was knocked down using shNodal which subsequently resulted in a reversal of CSC properties in A375 CSCs in regards to the expression of CSC markers. ALK4/5/7 blockage using SB431542 presented similar effects on CSC properties of A375 CSCs with Nodal knockdown, revealing that Nodal signaling was necessary for regulating A375 CSC features.

Nodal is a member of the TGF- $\beta$ superfamily of secreted proteins that signals through the serine/threonine kinase receptor family triggering the phosphorylation of Smad2 and $3(6,7)$. It is worth testing the regulation of Nodal induced by hypoxia on TGF- $\beta$ in further studies. It was reported that Nodal protein expression is positively correlated with HK-II, Glut-I and PDK-1 via activation of HIF-1 $\alpha(24,25)$. Consistently, in the present study, it is found that, Nodal upregulated by hypoxia exposure promoted HK-II, Glut-1, PDK-1 and HIF-1 $\alpha$ in A375 CSCs. Knockdown of Nodal remarkably attenuated hypoxic-induced upregulation of glycolysis-associated genes, indicating the critical role of Nodal in controlling glycolysis. By promoting glycolysis, hypoxic-induced Nodal prevented ROS accumulation and thus exerted protective effects on A375 CSCs. Instead of imaging ROS staining in spheres, we quantified ROS accumulation due to technical difficulty. However, it is still unknown how Nodal regulates this process.

Nodal is widely known as an inducer for mesendogermal genes and gastrulation movements within the epiblast after being activated and secreted after cleavage $(32,39)$. It was also found that uncleaved Nodal (proNodal) governs the expression of downstream target genes, including sonic hedgehog (shh) and FGFR3 transcriptionally or post-transcriptionally, indicating that proNodal is potentially involved in physiological regulation (15). Processed Nodal activates Nodal/Activin signaling to maintain quiescence and chemoresistance in melanoma cancer stem cells (40), however, whether proNodal takes part in these processes remains unknown. In the present study, introduction of mut-proNodal desensitized A375 CSCs pretreated with $10 \mu \mathrm{M} \mathrm{SB} 431542$, indicating that it functions independent of the ALK4/7 receptor. This was further confirmed by exposure of A375 CSCs to purified rhNodal or mut-proNodal, in which mut-proNodal failed to influence the chemosensitivity of the A375 CSCs to dacarbazine. By performing apoptotic analysis, introduction of mut-proNodal significantly induced desensitization of A375 CSCs to dacarbazine, and revealed it functions intracellularly independent with cell surface receptor ALK4/7.

In conclusion, this study provides novel information concerning the effects of hypoxia exposure on Nodal expression and functions in A375 CSCs. Our results revealed that hypoxia exposure upregulated Nodal expression and activated Nodal signal on Smad2/3, contributed to maintain stemness and promotes malignanct potential, including invasion, sphere formation and colony formation. Importantly, uncleaved Nodal precursor, proNodal, desensitized A375 CSCs to dacarbazine, without disturbing self-renewal capacity and sphere formation, independent of mature Nodal receptor ALK4/7. Further research is required to reveal the novel mechanism. Taken together, we revealed that hypoxia-induced Nodal promoted malignant behavior and chemoresistance in melanoma cancer cell A375.

\section{Acknowledgements}

The authors thank Ms H.M. Shi for English editing.

\section{Funding}

No funding was received.

\section{Availability of data and materials}

The datasets used during the present study are available from the corresponding author upon reasonable request.

\section{Authors' contributions}

LH and CY conceived and designed the study. LH, CJJ, WX, $\mathrm{HE}$ and ZZY performed the experiments. $\mathrm{LH}$ wrote the manuscript. CY reviewed and edited the manuscript. All authors read and approved the manuscript and agree to be accountable for all aspects of the research in ensuring that the accuracy or integrity of any part of the work are appropriately investigated and resolved.

\section{Ethics approval and consent to participate}

Cell lines were used in the study. No human tissues were used nor animal studies were carried out and thus no ethical approval was required.

\section{Consent for publication}

Not applicable.

\section{Competing interests}

All authors declare that they have no conflict of interests. 


\section{References}

1. Miller AJ and Mihm MC: Melanoma. N Engl J Med 355: 51-65, 2006.

2. Godar DE: Worldwide increasing incidences of cutaneous malignant melanoma. J Skin Cancer 2011: 858425, 2011.

3. Reed KB, Brewer JD, Lohse CM, Bringe KE, Pruitt CN and Gibson LE: Increasing incidence of melanoma among young adults: An epidemiological study in Olmsted County, Minnesota. Mayo Clin Proc 87: 328-334, 2012.

4. Bedia C, Casas J, Andrieu-Abadie N, Fabriàs G and Levade T: Acid ceramidase expression modulates the sensitivity of A375 melanoma cells to decarbazine. J Biol Chem 286: 28200-28209, 2011.

5. Mouawad R, Sebert M, Michels J, Bloch J, Spano JP and Khayat D: Treatment for metastatic malignant melanoma: Old drugs and new strategies. Crit Rev Oncol Hematol 74: 27-39, 2010.

6. Brennan J, Norris DP and Robertson EJ: Nodal activity in the node governs left-right asymmetry. Genes Dev 16: 2339-2344, 2002.

7. Takenaga M, Fukumoto M and Hori Y: Regulated nodal signaling promotes differentiation of the definitive endoderm and mesoderm from ES cells. J Cell Sci 120: 2078-2090, 2007.

8. Topczewska JM, Postovit LM, Margaryan NV, Sam A, Hess AR, Wheaton WW, Nickoloff BJ, Topczewski J and Hendrix MJ: Embryonic and tumorigenic pathways converge via Nodal signaling: Role in melanoma aggressiveness. Nat Med 12: 925-932, 2006.

9. Lee CC, Jan HJ, Lai JH, Ma HI, Hueng DY, Lee YC, Cheng YY, Liu LW, Wei HW and Lee HM: Nodal promotes growth and invasion in human gliomas. Oncogene 29: 3110-3123, 2010.

10. Lawrence MG, Margaryan NV, Loessner D, Collins A, Kerr KM, Turner M, Seftor EA, Stephens CR, Lai J, APC BioResource, et al: Reactivation of embryonic nodal signaling is associated with tumor progression and promotes the growth of prostate cancer cells. Prostate 71: 1198-1209, 2011.

11. Quail DF, Zhang G, Walsh LA, Siegers GM, Dieters-Castator DZ, Findlay SD, Broughton H,Putman DM, Hess DA and Postovit LM: Embryonic morphogen nodal promotes breast cancer growth and progression. PLoS One 7: e48237, 2012.

12. Papageorgiou I, Nicholls PK, Wang F, Lackmann M, Makanji Y, Salamonsen LA, Robertson DM and Harrison CA: Expression of nodal signalling components in cycling human endometrium and in endometrial cancer. Reprod Biol Endocrinol 7: 122, 2009.

13. Lonardo E, Hermann PC, Mueller MT, Huber S, Balic A Miranda-Lorenzo I,Zagorac S, Alcala S, Rodriguez-Arabaolaza I, Ramirez JC, et al: Nodal/Activin signaling drives self-renewal and tumorigenicity of pancreatic cancer stem cells and provides a target for combined drug therapy. Cell Stem Cell 9: 433-446, 2011.

14. Fuerer C, Nostro MC and Constam DB: Nodal-Gdf1 heterodimers with bound prodomains enable serum-independent nodal signaling and endoderm differentiation. J Biol Chem 289: 17854-17871, 2014.

15. Ellis PS, Burbridge S, Soubes S, Ohyama K, Ben-Haim N, Chen C, Dale K, Shen MM, Constam D and Placzek M: ProNodal acts via FGFR 3 to govern duration of Shh expression in the prechordal mesoderm. Development 142: 3821-3832, 2015.

16. Cabarcas SM, Mathews LA and Farrar WL: The cancer stem cell niche-there goes the neighborhood? Int J Cancer 129: 2315-2327, 2011.

17. Hanahan D and Weinberg RA: Hallmarks of cancer: The next generation. Cell 144: 646-674, 2011.

18. Zhu H, Wang D, Liu Y, Su Z, Zhang L, Chen F, Zhou Y, Wu Y, Yu M, Zhang Z and Shao G: Role of the Hypoxia-inducible factor-1 alpha induced autophagy in the conversion of non-stem pancreatic cancer cells into $\mathrm{CD} 133^{+}$pancreatic cancer stem-like cells. Cancer Cell Int 13: 119, 2013.

19. Quail DF, Taylor MJ, Walsh LA, Dieters-Castator D, Das P, Jewer M, Zhang G and Postovit LM: Low oxygen levels induce the expression of the embryonic morphogen Nodal. Mol Biol Cell 22: 4809-4821, 2011

20. Zhou S, Kurt-Jones EA, Cerny AM, Chan M, Bronson RT and Finberg RW: MyD88 intrinsically regulates CD4 T-cell responses. J Virol 83: 1625-1634, 2009.
21. dos Santos SC, Tenreiro S, Palma M, Becker J and Sá-Correia I: Transcriptomic profiling of the Saccharomyces cerevisiae response to quinine reveals a glucose limitation response attributable to drug-induced inhibition of glucose uptake. Antimicrob Agents Chemother 53: 5213-5223, 2009.

22. Walsh MC, Smits HP, Scholte M and van Dam K: Affinity of glucose transport in Saccharomyces cerevisiae is modulated during growth on glucose. J Bacteriol 176: 953-958, 1994.

23. Constam DB and Robertson EJ: Regulation of bone morphogenetic protein activities by prodomains and proprotein convertases. J Cell Biol 144: 139-149, 1999.

24. Vo BT and Khan SA: Expression of nodal and nodal receptors in prostate stem cells and prostate cancer cells: Autocrine effects on cell proliferation and migration. Prostate 71: 1084-1096, 2011.

25. Lai JH, Jan HJ, Liu LW, Lee CC, Wang SG, Hueng DY, Cheng YY, Lee HM and Ma HI: Nodal regulates energy metabolism in glioma cells by inducing expression of hypoxia-inducible factor 1 $\alpha$. Neuro Oncol 15: 1330-1341, 2013.

26. Papandreou I, Cairns RA, Fontana L, Lim AL and Denko NC: HIF-1 mediates adaptation to hypoxia by actively downregulating mitochondrial oxygen consumption. Cell Metab 3: 187-197, 2006.

27. Wang F, Zhang GY, Xing T, Lu ZY, Li JH, Peng C, Liu GH and Wang NS: Renalase contributes to the renal protection of delayed ischaemic preconditioning via the regulation of hypoxia-inducible factor-1 $\alpha$. J Cell Mol Med 19: 1400-1409, 2015.

28. Jian SL, Chen WW, Su YC, Su YW, Chuang TH, Hsu SC and Huang LR: Glycolysis regulates the expansion of myeloid-derived suppressor cells in tumor-bearing hosts through prevention of ROS-mediated apoptosis. Cell Death Dis 8: e2779, 2017.

29. Elliott RL and Blobe GC: Role of transforming growth factor beta in human cancer. J Clin Oncol 23: 2078-2093, 2005.

30. Glasgow E and Mishra L: Transforming growth factor-beta signaling and ubiquitinators in cancer. Endocr Relat Cancer 15: 59-72, 2008.

31. Beck S, Le Good JA, Guzman M, Ben Haim N, Roy K, Beermann F and Constam DB: Extraembryonic proteases regulate nodal signaling during gastrulation. Nat Cell Biol 4: 981-985, 2002.

32. Ben-Haim N, Lu C, Guzman-Ayala M, Pescatore L, Mesnard D, Bischofberger M, Naef F, Robertson EJ and Constam DB: The nodal precursor acting via activing receptors induces mesoderm by maintaining a source of its convertases and BMP4. Dev Cell 11: 313-323, 2006.

33. Eimon PM and Harland RM: Effects of heterodimerization and proteolytic processing on derriere and nodal activity: Implications for mesoderm induction in xenopus. Development 129: 3089-3103, 2002.

34. Strizzi L, Postovit LM, Margaryan NV, Seftor EA, Abbott DE, Seftor RE, Salomon DS and Hendrix MJ: Emerging roles of nodal and Cripto-1: From embryogenesis to breast cancer progression. Breast Dis 29: 91-103, 2008.

35. Shen MM: Nodal signaling: Developmental roles and regulation. Development 134: 1023-1034, 2007.

36. Duan W, Li R, Ma J, Lei J, Xu Q, Jiang Z, Nan L, Li X, Wang Z, Huo $X$, et al: Overexpression of nodal induces a metastatic phenotype in pancreatic cancer cells via the Smad2/3 pathway. Oncotarget 6: 1490-1506, 2015.

37. Strizzi L, Postovit LM, Margaryan NV, Lipavsky A, Gadiot J, Blank C, Seftor RE, Seftor EA and Hendrix MJ: Nodal as a biomarker for melanoma progression and a new therapeutic target for clinical intervention. Expert Rev Dermatol 4: 67-78, 2009.

38. Postovit LM, Margaryan NV, Seftor EA, Kirschmann DA, Lipavsky A, Wheaton WW, Abbott DE, Seftor RE and Hendrix MJ: Human embryonic stem cell microenvironment suppresses the tumorigenic phenotype of aggressive cancer cells. Proc Natl Acad Sci USA 105: 4329-4334, 2008.

39. Brennan J, Lu CC, Norris DP, Rodriguez TA, Beddington RS and Robertson EJ: Nodal signaling in the epiblast patterns the early mouse embryo. Nature 411: 965-969, 2001.

40. Cioffi M, Trabulo SM, Sanchez-Ripoll Y, Miranda-Lorenzo I, Lonardo E, Dorado J, Reis Vieira C, Ramirez JC, Hidalgo M, Aicher A, et al: The miR-17-92 cluster counteracts quiescence and chemoresistance in a distinct subpopulation of pancreatic cancer stem cells. Gut 64: 1936-1948, 2015. 